\title{
Pyogenic Granuloma of Lower Lip: An Unusual Presentation
}

\author{
Amita Rai, ${ }_{1}^{1}$ Bandana Koirala, ${ }^{2}$ Mamta Dali, ${ }^{3}$ Sneha Shrestha, ${ }^{4}$ Ashish Shrestha ${ }^{5}$ \\ ${ }^{1}$ Lecturer, ${ }^{2}$ Professor, ${ }^{3}$ Associate Professor, ${ }^{4}$ Assistant Professor, ${ }^{5}$ Additional Professor \\ ${ }^{I}$ Department of Pediatric and Preventive Dentistry, People's Dental College and Hospital, Nayabazar, Kathmandu, Nepal, \\ ${ }^{2-4}$ Department of Pedodontics and Preventive Dentistry, ${ }^{5}$ Department of Oral Pathology, College of Dental Surgery, B.P. Koirala Institute of \\ Health Sciences, Dharan, Sunsari, Nepal.
}

\begin{abstract}
Pyogenic granuloma is a benign inflammatory hyperplasia of connective tissue. It depicts an overzealous tissue response to a known stimulus or injury and is commonly found in the gingiva, especially in the anterior maxilla, and rarely on lips, oral mucosa, and tongue. It is commonly seen during the second,third,and fourth decade in females,with predilection in the third decade. This case report depicts the unusual presentation of pyogenic granuloma of the lower lip in an 11-year-old male child following a positive history of trauma and lip biting,which was successfully managed by excision under local anesthesia.
\end{abstract}

Keywords: Inflammatory hyperplasia, lower lip, pyogenic granuloma.

\section{INTRODUCTION}

Pyogenic granuloma (PG) was first described as "human botryomycosis" by Poncet and Dorin in 1897. Then the term "Pyogenic granuloma" (PG) was coined by Hartzell in $1904 .^{1}$ However some authors claim that the present name was given by Crocker in $1903 .^{2}$ It is a benign inflammatory hyperplasia of connective tissue. The term PG has been widely considered a misnomer because it neither produces pus nor represents a granulomatous inflammatory process. It is also known by other names such as; "eruptive hemangioma," "granulation tissue type hemangioma," "granuloma gravidarum," "lobular capillary hemangioma," "pregnancy tumor," "Crocker and Hartzell's disease," "vascular epulis," "benign vascular tumor," "hemangiomatosis granuloma," and "epulis teleangiectatium granulomatosa." ${ }^{1}$ Pyogenic granuloma is commonly found in the gingiva $(75 \%) .{ }^{3,4}$ The extra gingival lesions are quite uncommon,mimicking other benign soft tissue growths or even malignancy,when present. ${ }^{5}$

\section{Correspondence}

\section{Dr. Amita Rai}

Department of Pediatric and Preventive Dentistry,

People's Dental College and Hospital,Nayabazar,

Kathmandu,Nepal.

E-mail: amitarai2013@gmail.com

\section{Citation}

Rai A, Koirala B, Dali M, Shrestha S, Shrestha A. Pyogenic Granuloma of Lower Lip: An Unusual Presentation. J Nepal Assoc Pediatr Dent. 2021;2(1):24-7.

\section{CASE REPORT}

An 11-year-old male child presented to the department of Pedodontics and Preventive Dentistry, BPKIHS, Dharan with the chief complain of growth in the midline of the lower lip since two weeks. Positive history of trauma was found one month back along with lip-biting habit.

Intraoral examination revealed a well-circumscribed, single, pedunculated, erythematous mass of size $5 \times 5$ x $5 \mathrm{~mm} 3$ (Figure 1). On palpation, the lesion was firm in consistency and non-tender. Sign of hemorrhage on irritation was present. There were no signs of discharge, and visible or palpable pulsation. Based on the clinical findings, a provisional diagnosis of Irritational Fibroma was made,and an excisional biopsy was planned.

Informed consent from the parents and assent from the patient was taken for the procedure. The patient was subjected to hematological investigations wherein all values were within the normal ranges. The mass was excised from the base using surgical blade no. 15,followed by primary tissue closure with $4-0$ silk suture, and was sent for biopsy (Figure 2,3). The patient was also motivated to stop the lip-biting habit. Adequate healing was seen in one week,two weeks, and one month follow-up (Figure 4).

Histopathological examination revealed the following findings which also mimicked Lobular capillary hemangioma; para-keratotic hyperplastic stratified 


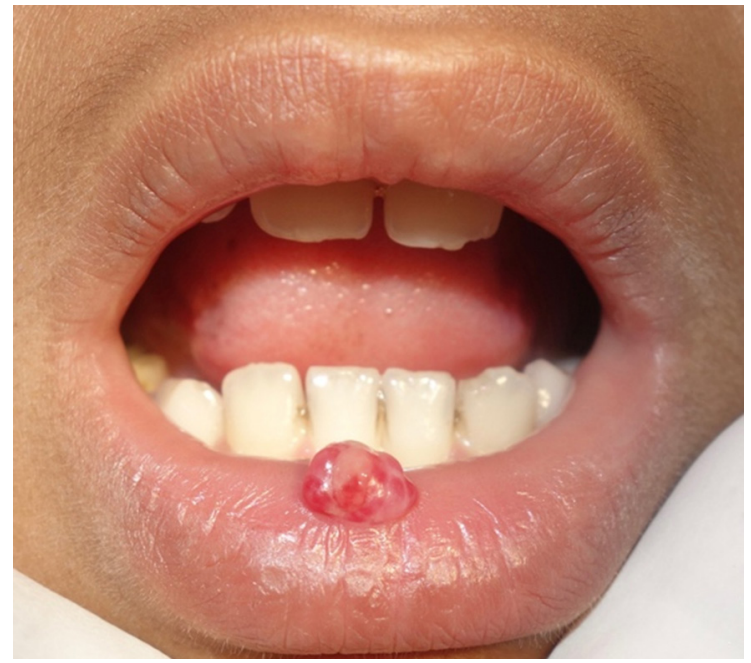

Figure 1. Pre-operative photograph.

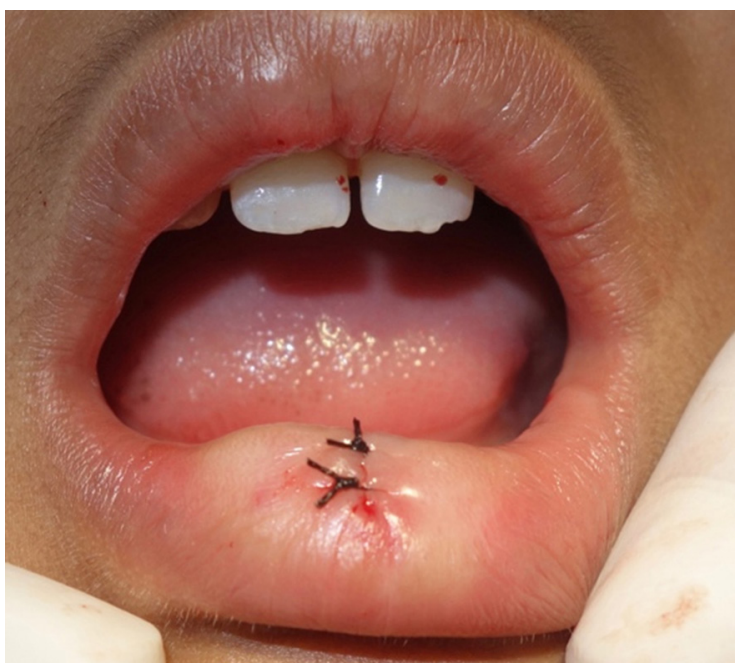

Figure 2. Immediate Post-operative photograph.

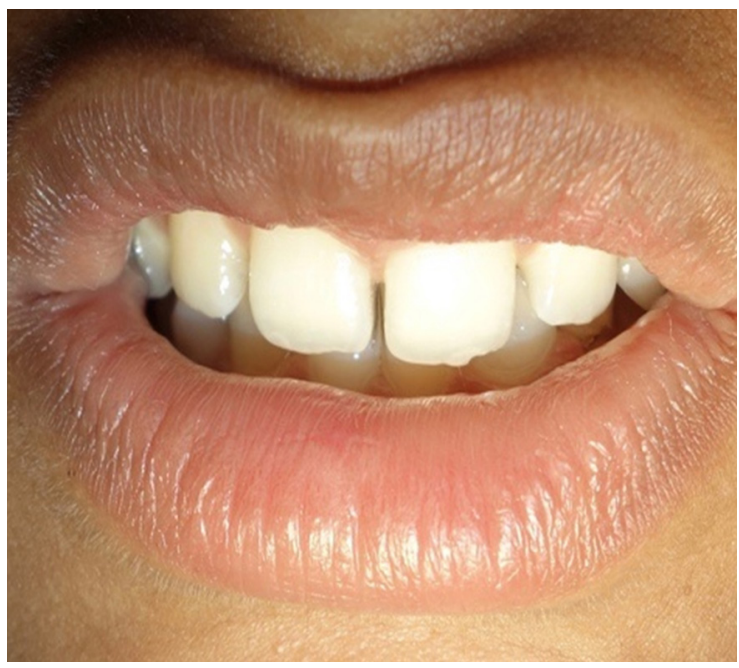

Figure 4. One-month follow-up.

squamous epithelium with irregular rete ridges, fibrous underlying connective tissue stroma comprising of dense aggregates of plump fibroblasts,numerous endothelial cell lined blood vessels along with extravasated RBCs with

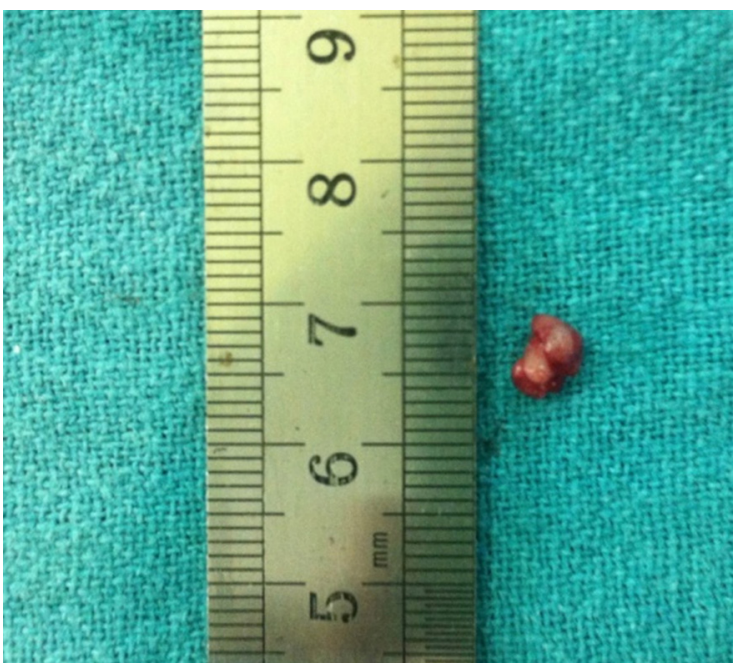

Figure 3. Excised mass.

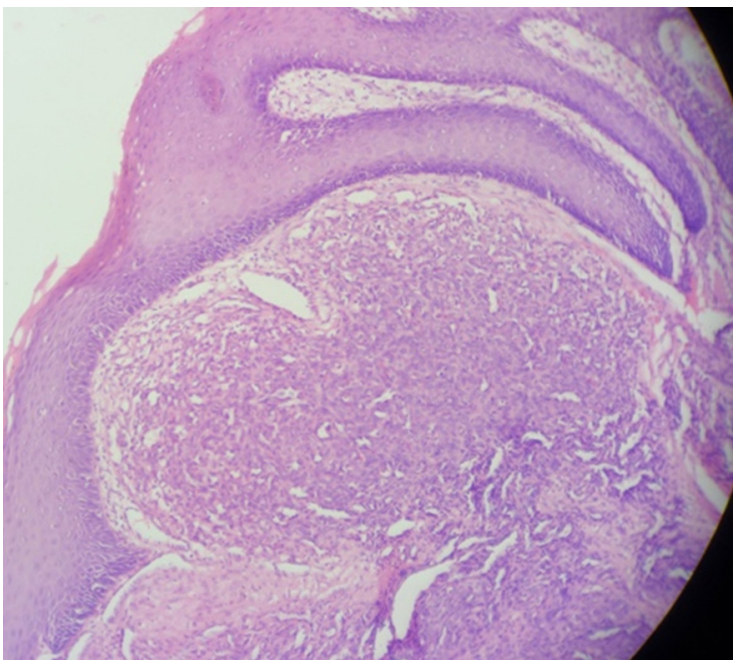

Figure 5. Histopathology image (10X,H\&E).

dense chronic inflammatory cell infiltration predominantly lymphocytes and plasma cells (Figure 5). Based upon the above findings along with clinical correlation, a diagnosis of Pyogenic Granuloma was made. 


\section{DISCUSSION}

In a study done by Saravana et. al, ${ }^{3}$ among 655 tumor-like lesions of the oral cavity, 137 lesions were confirmed as PG histologically,showing an incidence of $21 \%$. PG tends to show a marked predilection for the gingiva. Maxillary anterior labial gingiva is the commonest site of occurrence in both genders. ${ }^{3}$ PG can also be seen in other extraoral sites like hands, lips; uncommon sites are vulva, penis, esophagus,gut,and tracheobronchial tree. Gastrointestinal PG can cause severe anemia. ${ }^{5}$ It is commonly seen during the second,third, and fourth decade in females, due to the increased levels of circulating estrogen and progesterone hormones. ${ }^{1,3}$ In the present case, the occurrence was unusual with respect to age,gender and location.

Pyogenic granuloma might commonly result due to exaggerated localized connective tissue reaction to a minor tissue injury or underlying irritation. Calculus, poor oral hygiene, non-specific infection, overhanging restorations, cheek biting, etc. are considered as the irritating factors. ${ }^{1,3}$ Due to this irritation, the underlying fibrovascular connective tissue becomes hyperplastic, followed by proliferation of granulation tissue which leads to the formation of a PG. Factors such as inducible nitric oxide synthase, vascular endothelial growth factor, or connective tissue growth factor are known to be involved in angiogenesis and rapid growth of PG. ${ }^{1}$ Some authors also stated that oral PG arises as a result of some minor trauma to the tissues that provide a pathway for invasion of non-specific types of microorganisms. The tissues respond characteristically to these organisms of low virulence by the overzealous proliferation of a vascular type of connective tissue. ${ }^{2}$ PG has been found to be associated with certain medications such as oral contraceptives, retinoids, gefitinib, cabecitabine and afatinib. ${ }^{5}$ In the present case, presence of the lesion can be attributed to trauma as there was a positive history of trauma due to fall and lip-biting habit.

Pyogenic granuloma is also referred to as "pregnancy tumor", as it is seen during pregnancy especially during the second and third trimesters. Increased levels of estrogen and progesterone modify the vascular response to local irritants which leads to the occurrence of PG. ${ }^{6}$

The lesion can be described as early, established, and healing type, and the color of the lesion may also vary depending upon the clinical course. ${ }^{6}$ The early lesions may appear as pinkish and resemble the normal mucosal color, established lesions appear as reddish to purplish due to the increased vascularity and the healing lesions are usually seen as pinkish to whitish in color. The natural course of PG can also be categorized into three distinct phases; cellular phase,capillary phase/vascular phase and involutionary phase. ${ }^{6}$

The histopathological picture of PG shows exuberant granulation tissue which is covered by atrophic/ hyperplastic epithelium that may be ulcerated at times and reveals fibrinous exudates. The Presence of numerous endothelium-lined vascular spaces and proliferation of fibroblasts and budding endothelial cells are the characteristic features of pyogenic granuloma. The presence of mixed inflammatory cells infiltration is also observed. Cawson et al. ${ }^{7}$ have described two variants of PG depending on the rate of proliferation and vascularity, namely; lobular capillary hemangioma (LCH) and non-lobular capillary hemangioma (non$\mathrm{LCH})$. However, it should be remembered that these terms ( $\mathrm{LCH}$, non-LCH) have been used to describe PG based on its histopathological variations only, and it is not a true hemangioma in the real sense. The $\mathrm{LCH}$ type of pyogenic granuloma is characterized by proliferating blood vessels organized in lobular aggregates as seen in the present case, whereas the non-LCH type shows high vascular proliferation resembling granulation tissue. ${ }^{6,7} \mathrm{PG}$ can mimic other vascular tumors, including Kaposi form hemangioendothelioma, infantile hemangiomas, vascular malformations and Kaposi sarcoma. ${ }^{5}$ The term Lobular capillary hemangioma however is increasingly gaining favor in the dermatologic literature. ${ }^{2}$

The common treatment for PG consists of excision, which is also considered as the treatment modality with the lowest rate of recurrence. Depending on the site,size, and patient wishes; curettage, electrocautery, radiosurgery, cryosurgery (liquid nitrogen spray or a cryoprobe), sclerotherapy, or laser ( $\mathrm{Nd}$ : YAG, $\mathrm{CO}_{2}$ and flash lamp pulsed dye lasers) are alternative choices. ${ }^{5,8}$ Studies have reported a recurrence rate of up to $15.8 \%$ after conservative excision. ${ }^{2}$ Studies have also shown that lesions of gingival sites show a higher recurrence rate than lesions from other oral mucosal sites,after simple excision. In pregnant patients, the recurrence rate is more common. ${ }^{2,3}$ In the 
present case,no recurrence of the lesion was seen during the subsequent visits of one week, two weeks, and one month after the excision.

\section{CONGLUSIONS}

Pyogenic granuloma developing at extra-gingival sites can prove to be a diagnostic challenge to the clinician. This accentuates the importance of thorough history and clinical examination in the formulation of a diagnosis. However, histopathological examination is necessary to establish a final diagnosis. Cessation of etiologies and regular follow-up can prevent recurrence of such lesions.

Conflict of Interest: None

\section{REFERENGES}

1. Newadkar UR,Khairnar S,Dodamani A. Pyogenic granuloma: A clinicopathological analysis of fifty cases. J Oral Res Rev. 2018 Jun;10(1):7-10. [Full Text | DOI]

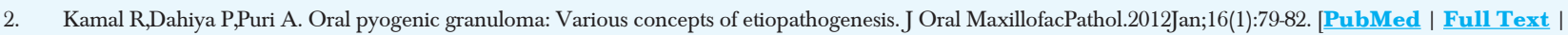
DOI]

3. Saravana GH. Oral pyogenic granuloma: a review of 137 cases. Br J Oral Maxillofac Surg. 2009 Jun;47(4):318-9. [ublMed | DOI]

4. Amirchaghmaghi M,Falaki F,Mohtasham N,Mozafari PM. Extragingival pyogenic granuloma: a case report. Cases J. 2008 Dec 3;1(1):371. [PulbMed | DOI]

5. Wollina U,Langner D,França K, Gianfaldoni S,Lotti T,Tchernev G. Pyogenic Granuloma - A Common Benign Vascular Tumor with Variable Clinical Presentation: New Findings and Treatment Options. Open Access Maced J Med Sci. 2017 Jul 13;5(4):423-426. [uㅣMed I DOI]

6. Marla V,Shrestha A,Goel K,Shrestha S. The Histopathological Spectrum of Pyogenic Granuloma: A Case Series. Case Rep Dent. 2016;2016:1323798. [PubMed I DOI]

7. Cawson RA,Binnie WH,Speight P,Barrett AW,Wright JM. Lucas's Pathology of tumors of the oral tissues,5th edition. London; Churchill Livingstone,1998. [Link]

8. Rizwanulla M,Koirala B,Sharma S,Adhikari L,Pradhan A. Pyogenic Granuloma: A Case Report. Health Renaissance. 2010 Dec;8(3):196-8. [피ll Text | DOI] 